\title{
Predictions on the core mass of Jupiter and of giant planets in general
}

Accepted for publication in Astrophysics \& Space Science

\author{
Nadine Nettelmann
}

\begin{abstract}
More than 80 giant planets are known by mass and radius. Their interior structure in terms of core mass, number of layers, and composition however is still poorly known. An overview is presented about the core mass $M_{\text {core }}$ and envelope mass of metals $M_{Z}$ in Jupiter as predicted by various equations of state. It is argued that the uncertainty about the true $\mathrm{H} / \mathrm{He} \mathrm{EOS}$ in a pressure regime where the gravitational moments $J_{2}$ and $J_{4}$ are most sensitive, i.e. between 0.5 and $4 \mathrm{Mbar}$, is in part responsible for the broad range $M_{\text {core }}=0-18 M_{\oplus}, M_{Z}=0-38 M_{\oplus}$, and $M_{\text {core }}+M_{Z}=14-38 M_{\oplus}$ currently offered for Jupiter. We then compare the Jupiter models obtained when we only match $J_{2}$ with the range of solutions for the exoplanet GJ 436b, when we match an assumed tidal Love number $k_{2}$ value.
\end{abstract}

Keywords giant planets: interior; individual: Jupiter

\section{Introduction}

Jupiter is the best studied giant planet. Its oblate shape and the corresponding elliptic gravity field deformation have been measured within an accuracy of $10^{-4}$. The uncertainty of its mass reflects the uncertainty of the gravitational constant $G$, and even its atmospheric He abundance is known within 3\% (see Guillot and Gautier 2007 for an overview). However, despite several spacecraft missions to Jupiter and observational data sampling over several centuries, we even do not know whether Jupiter possesses a core or

Nadine Nettelmann

University of California, Santa Cruz, Dept. of Astronomy and Astrophysics, CA 95064, USA

Universität Rostock, Institut für Physik, D-18051 Rostock, Germany not (Saumon and Guillot 2004), and if heavy elements in the interior are distributed homogeneously. These are fundamental interior structure properties. If we are to describe the interior of Jupiter and other giant planets, we shape our imagination of interior structure into terms such as core mass and envelope metallicity and apply the simplest possible structure type that can quantitively answer our questions. For instance, the assumption of a completely homogeneous interior is sufficient to explain the observed mass and radius of a extrasolar giant planet, and an occasional additional constraint can be satisfied by assuming the presence of a core within a two-layer approach. For solar giant planets however, a two-layer approach is not consistent with available observational constraints when non-empirical equations of state are applied (e.g.; see Chabrier et al. (1992) for Jupiter and Saturn, and Helled et al. (2010) for Uranus and Neptune), and hence we here model the interior by assuming three layers. This article is not about the difficulties we run into by relying on this approach. It is about the resulting core mass and metallicity of Jupiter (\$3.1), where we repeat some of the analyis published in Fortney and Nettelmann (2010) (hereafter FN10). We then continue in $\S 3.2$ to investigate the specific open question if the assumption of an homogeneous heavy element dstribution can be subject to free choice in the case of recently published ab-initio data based Jupiter models, as it has been suggested in Militzer and Hubbard (2009). In ( $\S$ (4) we show the degeneracy of solutions raised by ignoring the octupole moment of the gravity field, $J_{4}$ equivalent to knowing the Love number $k_{2}$ of an extrasolar planet, but not $k_{4}$ 


\section{Modeling giant planets in isolation}

\subsection{Observational constraints}

Interior models should reproduce the observed parameters. These are, first of all, mass $M_{p}$ and radius $R_{p}$ of the planet. For Jupiter, Saturn, and Uranus, the radius is the observable equatorial radius $R_{e q}$ taken at the 1 bar pressure level. Below that the interior is adiabatic. For close-in extrasolar giant planets, the observed transit radius occurs at 4-6 orders of magnitude lower pressures than the onset of the adiabatic region, and a proper outer boundary condition has to be provided by a model atmosphere. Calculations for particular giant planets predict the radiative/convective layer boundary deep in the atmosphere between 100 and 1000 bars (Showman et al. 2008). The mean helium abundance should equal that of the protostellar cloud where the planet formed from. However, the atmospheres of Jupiter and Saturn are depleted in helium compared to the solar value $Y=0.270 \pm 0.005$ (Bahcall and Pinsonneaul 1995), which motivates the introduction of a He-poor outer envelope and a He-rich inner envelope. The metallicity $Z$ in the atmospheres of the solar planets is enhanced above the solar value $Z_{\odot}=0.015$ Lodders 2003) as derived from abundance measurements of single species (e.g.; C, N, S, Ar, Xe). Solar planets are fast rotators, which gives rise to a gravity field deformation as expressed by the gravitational moments $J_{2}, J_{4}$, and $J_{6}$. Equivalent constraints for extrasolar planets are tidal Love numbers $k_{2 n}$. Parameters related to thermal or orbital evolution can give additional constraints but are not addressed in this article.

\subsection{The Three-layer structure assumption}

For Jupiter and Saturn, the simplest structure type that is consistent with the constraints described above has three layers: a core of rocks and/or ices, and two envelopes that are convective, adiabatic, and homogeneous, but differ in the mass fraction of helium $(Y)$ and metals $(Z)$. We choose the metallicities $Z_{1}$ and $Z_{2} 1$ to adjust $\mathrm{J}_{2}$ and $J_{4}$. Often (e.g; Guillot 1999; Hori et al. 2008) but not always (Militzer et al. 2008) models that reproduce Jupiter's $J_{2}$ and $J_{4}$ are found to reproduce also $J_{6}$ within the obervational error bar. Physical reasons for a discontinuity in He or metals can be phase transitions, $\mathrm{H} / \mathrm{He}$ phase separation, and in particular

${ }^{1}$ Index 1 refers to the outer envelope, and index 2 to the inner envelope. for metals the process of planet formation, but its location is essentially unconstrained, and hence the transition pressure $P_{1-2}$ between the envelopes a free parameter. In the absence of these particular physical processes, gaseous planets are likely to have a homogenous envelope because of convection. If however convection is inhibited as suspected for Uranus (Podolak et al. 1991) a series of double-diffusive layers can develop creating a large-scale compositional gradient. Such complicated models are not considered here.

\subsection{Quasi-adiabat and equation of state}

At layer boundaries, pressure $P$ and temperature $T$ are required to transit continously. The $P-T-\rho$ profile, where $\rho$ is mass density, along the quasi-adiabat depends on the He abundances and metallicities chosen, and on the equations of state (EOS) of the underlying materials allowed for. If the envelopes differ in He mass fraction $\left(Y_{1}<Y_{2}\right)$ or metallicity $\left(Z_{1} \neq\right.$ $Z_{2}$ ), then entropy and density transit discontinously, so we call the internal $P-T$ profile quasi-adiabatic. At a layer boundary, a boundary layer can develop with change in temperature across or conductive heat transport, causing a warmer interior at higher entropy. The effect of a boundary layer has been included by Fortney and Hubbard (2003) for the inhomogeneous evolution of Saturn; but not yet in structure calculations. Since the gravity data probe the internal $P-\rho$ relation, the expected influence of a warmer interior on the structure would be a higher deep envelope metallicity.

It is convenient to represent elements heavier than He (metals) by an equation of state of water (e.g.; Fortney and Hubbard 2003; Saumon and Guillot 2004) as also supported by planet formation theory (Helled et al. 2008), and to assume the core be made of rocks (e.g.; Fortney and Hubbard 2003; Saumon and Guillot 2004; Nettelmann et al. 2008) or of water ice (e.g.; Saumon and Guillot 2004). Jupiter-sized giant planets are giant in size because they are predominantly composed of the light elements $\mathrm{H}$ and He. For details of the EOS, see Saumon and Guillot (2004); Nettelmann et al. (2008), FN10, and Militzer et al. (2008).

\subsection{Calculation of core mass and metallicity}

In order to obtain core mass and metallicity we integrate the 1-dimensional equations of mass conservation, $d m / d l=4 \pi l^{2} \rho(l)$, and of hydrostatic equilibrium,

$\frac{1}{\rho(l)} \frac{d P}{d l}=\frac{d U}{d l}$, 
along the quasi-adiabat for given outer boundary conditions and abundances of helium and metals. The coordinate $l$ parametrizes surfaces of constant total potential $U$. In spherical symmetry, i.e. in the absence of rotation, $l$ equals the radial coordinate $r$. The most often theory used to calculate the gravity field deformation according to shape deformation is the Theory of Figures by Zharkov and Trubitsyn (1978), but alternative theories can be applied as well (e.g.; Hubbard 1984). The gravity field deformation on or exterior to the surface $(\rho(P=1$ bar $) \approx 0)$ can be described by multipole expansion into spherical harmonics, where the expansion coefficients are the gravitational moments

$J_{2 n}=-\frac{1}{M_{p} R_{e q}^{2 n}} \int_{V} d^{3} r \rho(r, \theta) r^{2 n} P_{2 n}(\cos \theta)$.

They are intergrals over the internal mass density within the volume $V$ of the planet. Therefore, we can use the metallicities $Z_{1}$ and $Z_{2}$, which affect $\rho(l)$ along the quasi-adiabat, to adjust the moments $J_{2}$ and $J_{4}$. In general $Z_{1} \neq Z_{2}$. The core mass $M_{\text {core }}$ is then used to satisfy the inner boundary condition $m(l=0)=0$ for given $M_{p}\left(R_{p}\right)$.

\section{Jupiter}

3.1 Predicted core mass and envelope mass of metals

Figure 1 is a modified version of Fig. 1 in FN10 and shows model results for the core mass and envelope mass of metals of Jupiter predicted by different equations of state. These are DFT-MD2 (Militzer et al. 2008), LM-REOS based on FT-DFT-MD 3 with different EOS representing metals, namely $\mathrm{He}_{4}$ and $\mathrm{H}_{2} \mathrm{O}$ REOS (Nettelmann et al. 2008), SCvH-i and Sesamep (Guillot 1999; Saumon and Guillot 2004), Sesame-92 (the original Sesame-EOS for $\mathrm{H}$ and He (Lyon and Johnson 1992) together with $\mathrm{H}_{2} \mathrm{O}-\mathrm{REOS}$ ), and Sesame-K04 (a revised $\mathrm{H}$ and He Sesame-EOS with minor constituent EOSs from the Panda-Code; see Kerley 2004). The Jupiter models are published in the same references apart from the Sesame-92 based models, which were calculated in Nettelmann (2009). For each particular equation of state, numerous solutions are found due to uncertainties in the constraints applied. Obviously, a large uncertainty in Jupiter's core mass $\left(0-18 M_{\oplus}\right)$ and envelope metallicity $\left(0-38 M_{\oplus}\right)$ arises from the broad

${ }^{2}$ Density Functional Theory for the electrons and Molecular Dynamics simulations for the ions in the warm dense matter regime

${ }^{3}$ In addition to DFT-MD, inclusion of finite temperature effects on the electronic subsystem, which affect the forces onto the ions variety of currently competing hydrogen equations of state. The total mass of metals $M_{\text {core }}+M_{Z}$ is better constrained and amounts to $14-38 M_{\oplus}$ (more precisely, in the order of the above EOS listing: $14-24,20-24$ and $28-32,17-37,33,38$, and $35 M_{\oplus}$ ). Thus to our current knowledge $4.4-12 \%$ of Jupiter's mass are heavy elements, which is an enrichment factor of $3-8$ over solar metallicity.

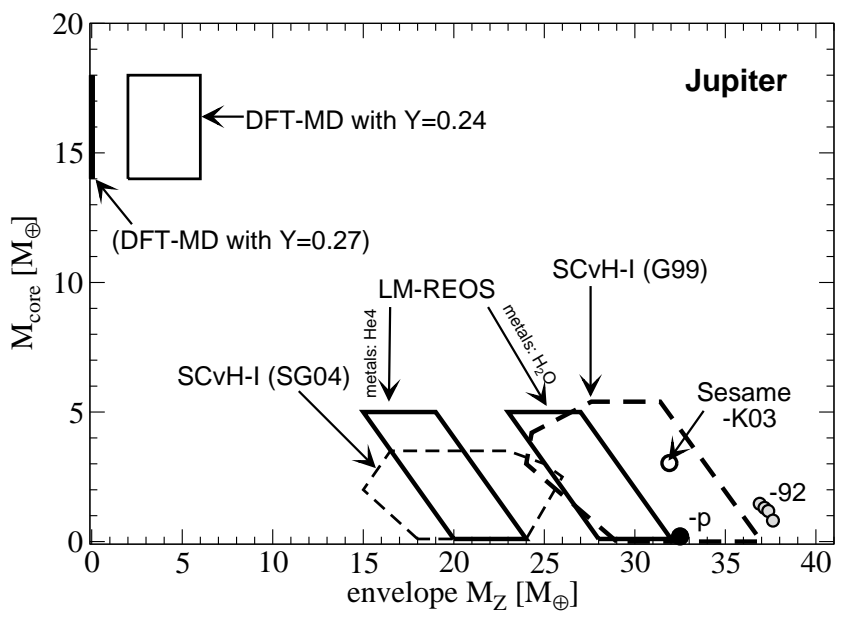

Fig. 1 Model results for the core mass $\left(M_{\text {core }}\right)$ and mass of metals $\left(M_{Z}\right)$ in the envelope(s) of Jupiter. Each polygon embraces the solutions found using a particular set of equations of state as labeled, for the components $\mathrm{H}, \mathrm{He}$, and metals. Models using $\mathrm{SCvH}-\mathrm{i}$ (SG04) and Sesame$\mathrm{p}$ assume $Z_{1}=Z_{2}$ and $Y_{1}<\bar{Y}<Y_{1}$ and are taken from Saumon and Guillot (2004), SCvH-i (3L) based models have $Z_{1} \neq Z_{2}$ (Guillot 1999), the Sesame-K04 model has $Z_{1}<Z_{2}$ Kerley 2004) as have LM-REOS based models (updated from Nettelmann et al. 2008), and the DFT-MD labeled model is a two-layer model with $Y_{1}=Y=0.24$ throughout the envelope. Adjusting this model for the protosolar mean value $Y=0.27$ by replacing $9 M_{\oplus}$ of metals by He yields a metal free envelope as indicated. This figure shows $0 \leq M_{\text {core }} \leq 18 M_{\oplus}, 0 \leq M_{Z} \leq 38 M_{\oplus}$, and $14 \leq M_{\text {core }}+M_{Z} \leq 38$, meaning that Jupiter's interior structure is not well known today and that models depend on the equation of state preferred.

Since core mass and envelope mass of metals are determined by the requirement to find internal density distributions that reproduce $M_{p}\left(R_{p}\right), J_{2}$ and $J_{4}$, the results reflect the compressibility of the $\mathrm{H} / \mathrm{He}$ subsystem along the quasi-adiabat. It is then particularly strange that similar methods used to calculate $\mathrm{H}$ and He EOS yield contrary results on $M_{\text {core }}$ and $M_{Z}$, as it is the case for DFT-MD and LM-REOS based on FT-DFT-MD (see Nettelmann et al. 2008; Holst et al. 2008; Kietzmann et al. 2007; French et al. 2009, for details). 
3.2 The freedom to assume a discontinuity in heavy elements

In $\S 3.1$, some of the models were based on the assumption $Z_{1}=Z_{2}$, while others (i.e. those using SCvH-i-99, Sesame-K03, Sesame-92, LM-REOS) were not. Here we show that this assumption is not for every EOS subject to free choice, if the model is required to meet $J_{2}, J_{4}$, and $J_{6}$. In particular, LM-REOS based Jupiter models require $Z_{1} \ll Z_{2}$, while DFT-MD based models $Z_{1} \approx Z_{2}$.

The convergent procedure used for LM-REOS based models is illustrated in Fig,2. Along the thick solid line, $Z_{1}=Z_{2}$. On that line, there is one model that matches $J_{2}$. However, $J_{4} / 10^{-4}=-6.04$ for this model is $8 \sigma$ afar from the observed $J_{4}$ value (see figure caption for details). Hence the convergent procedure follows the thin black line in the direction of the arrows until both $J_{2}$ and $J_{4}$ are met. Along that path, the core mass decreases from $12 M_{\oplus}$ down to $1.9 M_{\oplus}$. The converged solution has $Z_{1} \ll Z_{2}$. This behavior applies to every LM-REOS based Jupiter model, and hence the choice $Z_{1}=Z_{2}$ cannot be made unless the interior does not rotate rigidy but instead the observed $J_{4}$ value is influenced by deep winds (Militzer et al. 2008).

On the other hand, as Fig. 2 shows, $Z_{1} \ll Z_{2}$ is not a favorite option for the DFT-MD based Jupiter model, since that would imply $Z_{1} \ll Z_{\odot}$ or even zero. This would contradict heavy element abundance measurements in Jupiter's atmosphere, which indicate $Z_{1} \geq 2 \times Z_{\odot}$. Therefore, $Z_{1} \approx Z_{2}$ is not a free choice for the DFT-MD based model. Consequently, the very different Jupiter core masses obtained using these two ab-initio EOS are not primarily due to different assumptions about the distribution of heavy elements. In order to resolve this problem, a comparison of the $\mathrm{H} / \mathrm{He}$ adiabats is highly desirable. Furthermore, isentropic compression experiments in the $0.5-4$ Mbar regime where $J_{2}$ and $J_{4}$ are most sensitive to metallicty would greatly help to discriminate between competing Jupiter models. For the other EOS considered here, both the assumptions $Z_{1}=Z_{2}$ or $Z_{1} \neq Z_{2}$ give acceptable Jupiter models and hence are a matter of free choice.

\section{Extrasolar planet models}

\section{$4.1 J_{2}$ and $k_{2}$}

As we have seen in $\S[$ Jupiter models that use one particular EOS can have various resulting $\left\{M_{\text {core }}, Z_{1}, Z_{2}\right\}$ triples. Without the constraint imposed by $J_{4}$ however, the variety of solutions would be immense, including $M_{\text {core }}>18 M_{\oplus}$ and $M_{Z}>38 M_{\oplus}$. For extrasolar

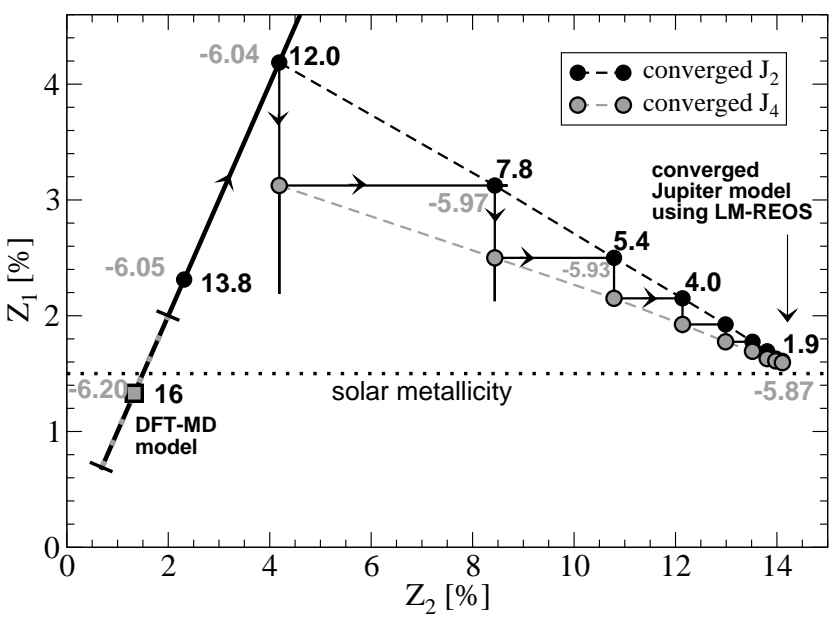

Fig. 2 Converged and intermediate envelope metallicities $Z_{1}, Z_{2}$ of a three-layer Jupiter model based on LMREOS. The converged model matches the most recent value $J_{4} / 10^{-4}$ value (grey numbers) of -5.87 (Jacobson 2003), has $M_{\text {core }}=1.9 M_{\oplus}$ (black numbers) and $Z_{1}=1.6 \%$. This model was obtained by an interative procedure that produced intermediate solutions along the solid black line. Starting from $Z_{1}=Z_{2}=1 \%$, the envelope metallicity is increased along the $Z_{1}=Z_{2}$ line of homogeneous metallicity (thick black solid) until $J_{2}$ is matched. Such a model with LM-REOS would have $M_{\text {core }}=12.0 M_{\oplus}$ and $J_{4} / 10^{-4}=-6.04$. Then iteratively $J_{4}$ and $J_{2}$ are fitted by allowing for $Z_{1} \neq Z_{2}$ as indicated respectively by grey circles and black circles until outer and inner envelope metallicities are found that match both $J_{2}$ and $J_{4}$ (converged model). All models along the dashed black line match $J_{2}$, and all models along the dashed grey line match $J_{4}$. This procedure cannot be applied to bring the $J_{4}$ value of the DFT-MD based Jupiter model (grey square with error bars) (Militzer et al. 2008 ) in agreement with the observed $J_{4}$ value, since obviously $Z_{1} \ll Z_{\odot}$ would rapidly occur.

planets, currently available gravity data are $M_{p}$ and $R_{p}$ only. A potentially observational constraint equivalent to $J_{2}$ has been suggested to be the tidal Love number $k_{2}$ (Ragozzine and Wolf 2009), which measures the ability of a planet to develop an elliptic deformation in response to a tidal perturber such as the close-by parent star. In this section, we present implications of knowing a precise $k_{2}$ value of the Neptune-sized extrasolar planet GJ 436b.

\subsection{Core mass of GJ 436b models for given $k_{2}$}

About 30 light years away from Earth, the Hot Neptune GJ $436 \mathrm{~b}\left(M_{p}=23.17 M_{\oplus}, R_{p}=4.22 R_{\oplus}\right)$ (Torres et al. 2008) orbits the M-star GJ436. Placed in a mass-radius diagram, this planet is located close to theoretical $M-R$ relations of warm water planets. Accordingly, interior structure models assuming an iron-silicate core, a water layer, and a $\mathrm{H} / \mathrm{He}$ envelope allow for a composition of 
$95 \%$ water and $5 \% \mathrm{H} / \mathrm{He}$ (Figueira et al. 2009), but also for a water-less composition. $\mathrm{H} / \mathrm{He}$ envelope models, whether dry or not, are found to have $0.02<k_{2}<0.2$ (Nettelmann et al. 2010). We here assume $k_{2}=0.2$ and search for the core mass and water content that satisfy this additional constraint. We allow water be mixed homogeneously into the $\mathrm{H} / \mathrm{He}$ envelope (twolayer model) or confined to a deep water layer (threelayer models) and combinations in between (Neptunelike three-layer models). Figure 3 shows the result in comparison with LM-REOS based Jupiter models forced to meet the observed $J_{2}$ value, but not $J_{4}$.
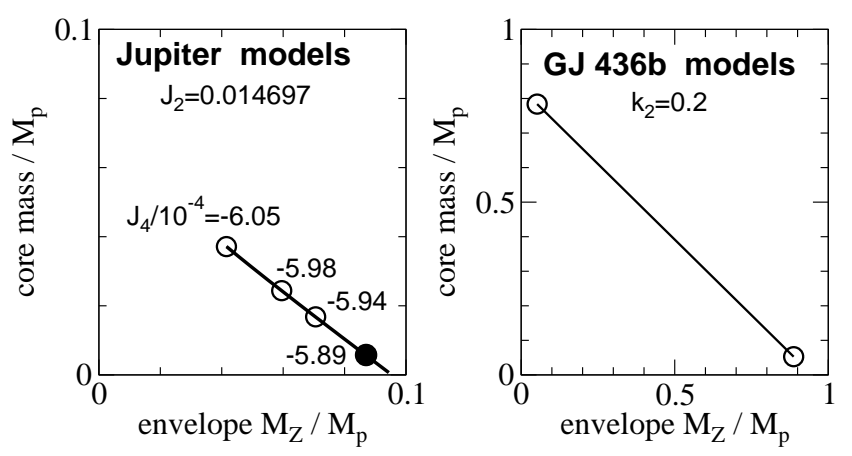

Fig. 3 Jupiter models matching the measured $J_{2}$ value, and GJ 436b models matching an arbitrary $k_{2}$ value. Maximum core mass (no water layer) and minimum core mass (no rock core) solutions are found. Accurate $(\sim 1 \%)$ knowledge of Jupiter's $J_{4}$ reduces the uncertainties in $M_{\text {core }}$ and $M_{Z}$ (filled circle solution), while GJ 436b's core mass is undetermined if $k_{2}=0.2$ and $k_{4}$ remains unknown.

In both cases, the solutions are located along a straight line (solutions for different $k_{2}$ or $J_{2}$ values would span parallel lines). For both planets, and hence in general, the upper limit in core mass is obtained for homogeneous envelope models (compare $\S(3.2)$, and the upper limit in envelope mass of metals (here water) by zero-mass core models. In between there are three-layer models with various metallicities in the two envelopes. As indicated for Jupiter, solutions below the filled circle are also in agreement with $J_{4}$.

We conclude that the constraint imposed by $k_{2}$ or $J_{2}$ reduces the degeneracy of models, but further constraints are needed to determine the core mass of a planet within a few percent of total mass (Jupiter: $6 \%$ ). Cooling curve calculations might help to constrain the composition, since they depend on specific heat which significantly varies between relevant bulk materials. Unfortunately, evolution calculations are subject to uncertainties such as tidal interactions Leconte et al. 2010) and stellar irradiation and the response of the atmosphere (e.g. Valencia et al. 2010).

\section{Conclusions}

Standard Jupiter interior models predict a core mass of $0-18 M_{\oplus}$, an envelope mass of metals of $0-38 M_{\oplus}$, and a total mass of metals of $14-38 M_{\oplus}$. These uncertainties have increased over the past ten years Guillot 1999), when they were thought to arise from the possible presence of a plasma phase transition in hydrogen. From the behavior of model solutions discussed in this article, we conclude an unsufficient understanding of the $\mathrm{H} / \mathrm{He} \mathrm{EOS}$ in the $0.5-4 \mathrm{Mbar}$ pressure regime, where $J_{2}$ and $J_{4}$ are most sensitive. Equivalent to Jupiter models, measuring the $k_{2}$ value of an extrasolar planet would reduce possible solutions to a linear relation between core mass and envelope mass of metals, as shown for GJ 436b.

The author kindly thanks R. Redmer, J.J. Fortney, T. Guillot, and D.J. Stevenson for insightful discussions, U. Kramm for providing the $k_{2}$ values, and the anonymous referee for valuable comments. 


\section{References}

Bahcall, J.N., Pinsonneault, M.H.: Rev. Mod. Phys. 67, 781 (1995)

Chabrier, G., Saumon, D., Hubbard, W., Lunine, J.: Astrophys. J. 391, 826 (1992)

Figueira, P., Pont, F., Mordasini, C., Alibert, Y., Gorgy, C., Benz, W.: Astron. Astrophys. 493, 671 (2009)

Fortney, J.J., Hubbard, W.B.: Icarus 164, 228 (2003)

Fortney, J.J., Nettelmann, N.: In: Planetary Magnetism. Springer Space Sci. Rev., vol. 157, 423 (2010)

French, M., Mattsson, T.R., Nettelmann, N., Redmer, R.: Phys. Rev. B 79, 954107 (2009)

Guillot, T.: Planet. Space Sci. 47, 1183 (1999)

Guillot, T., Gautier, D.: Treat. of Geophys. 10, 439 (2007)

Helled, R., Anderson, J.D., Podolak, M., Schubert, G.: Astrophys. J.(2010, in press)

Helled, R., Podolak, M., Koretz, A.: Icarus 195, 863 (2008)

Holst, B., Redmer, R., Desjarlais, M.P.: Phys. Rev. B 77, 184201 (2008)

Hori, Y., Sano, T., Ikoma, M., Ida, S.: In: Exoplanets: Detection, Formation and Dynamics, IAU Symposium proceedings, vol. 249, 163 (2008)

Hubbard, W.B.: Planetary Interiors. Van Nostrand Reinhold Company Inc. (1984)

Jacobson, R.A.: JUP230 orbit solution. (NASA-JPL document) (2003). http://ssd.jpl.nasa.gov/?gravity_fields_op

Kerley, G.: (2004). Kerley Tech. Res. Report KTS04-1

Kietzmann, A., Holst, B., Redmer, R., Desjarlais, M.P., Mattsson, T.R.: Phys. Rev. Lett. 98, 190602 (2007)

Leconte, J., Chabrier, G., Baraffe, I., Levrard, B.: A\&A 516, A64 (2010)

Lodders, K.: Astrophys. J. 591, 1220 (2003)

Lyon, S., Johnson, J.D.e.: SESAME: Los Alamos National Laboratory Equation of State Database. Technical report, LANL report no. LA-UR-92-3407 (1992)

Militzer, B., Hubbard, W.B.: Astrophys. Space Sci. 322, 129 (2009)

Militzer, B., Hubbard, W.B., Vorberger, J., Tamblyn, I., Bonev, S.B.: Astrophys. J. 688, L54 (2008)

Nettelmann, N.: (2009). PhD-Thesis; U Rostock

Nettelmann, N., Holst, B., Kietzmann, A., French, M., Redmer, R., Blaschke, D.: Astrophys. J. 683, 1217 (2008)

Nettelmann, N., Kramm, U., Redmer, R., Neuhäuser, R.: Astron. Astrophys. 523, A26 (2010)

Podolak, M., Hubbard, W., Stevenson, D.: In: Uranus. 29. U Arizona Press (1991)

Ragozzine, D., Wolf, A.S.: Astrophys. J. 698, 1778 (2009)

Saumon, D., Guillot, T.: Astrophys. J. 609, 1170 (2004)

Showman, A.P., Cooper, C.S., Fortney, J.J., Marley, M.s.: Astrophys. J. 682, 559 (2008)

Torres, G., Winn, J.N., Holman, M.J.: Astrophys. J. 677, 1324 (2008)

Valencia, D., Ikoma, M., Guillot, T., Nettelmann, N.: Astron. Astrophys. 516, A20 (2010)

Zharkov, V.N., Trubitsyn, V.P.: Physics of Planetary Interiors. Tucson: Parchart (1978)

This manuscript was prepared with the AAS LATEX macros v5.2. 